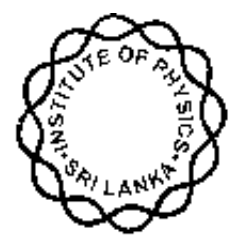

INSTITUTE OF PHYSICS - SRI LANKA

Research Article

\title{
First use of NMR diffusometry for the determination of core size distributions in core-shell latex films
}

\author{
P. Ekanayake ${ }^{a, *}$ and J.L. Keddie ${ }^{b}$ \\ ${ }^{\mathrm{a}}$ Department of Physics, University of Peradeniya, Peradeniya, Sri Lanka \\ ${ }^{\mathrm{b}}$ Department of Physics, University of Surrey, Guildford, Surrey GU2 7XH, UK
}

\begin{abstract}
Latex films cast from core-shell particles were characterised for the first time using restricted diffusion measurements obtained with a five-pulse steady-gradient stimulated echo (SGSE) nuclear magnetic resonance (NMR) technique in which all nuclear spin relaxation losses were kept constant. The latex particles, which were made via miniemulsion polymerization, consist of an acrylic shell and a poly(dimethyl siloxane) (PDMS) core. It was found that the particle core remains intact after film formation. The average sizes and distributions of the PDMS phases were determined. Unlike various microscopies, NMR diffusometry provides a powerful means for obtaining quantitative information about the average microstructure in an entire film.
\end{abstract}

\section{INTRODUCTION}

Latex particles in which a core of one polymer is surrounded by a shell of a second polymer are important in many different applications, including impact-resistant plastics and waterborne coatings and adhesives. The nanostructure of the individual particles, the interface between the core and shell polymers, and the sizes of both the core and the shell can each greatly alter the macroscopic properties of the final film [1].

It has been demonstrated that increasing the glass transition temperature $\left(T_{g}\right)$ of a core polymer, increases the elastic modulus of a film, whereas interparticle bonding can be used to adjust the strain at failure [2,3]. Clearly, the determination of the particle and film

\footnotetext{
* Corresponding author: Email: piyasiri2001@yahoo.co.uk Current address: Applied Physics Group, Faculty of Science, University of Brunei Darussalam, Jalan Tungku Link, Gadong BE1410, Negara Brunei Darussalam
} 
nanostructure is an important task in optimizing the structure/property relationship in coreshell latex materials.

The nanostructure of core-shell particles and films is usually characterised by transmission electron microscopy (TEM) [4], scanning electron microscopy (SEM) [5], atomic force microscopy (AFM), [6,7] and X-ray or neutron scattering [8,9]. Studies of films often require careful cross-sectioning and sample preparation to avoid the introduction of artefacts. In the study of individual particles, high resolution microscopies can provide information only about a relatively small number of particles (on the order of 100 ) at one time, even though a liter of a $50 \mathrm{wt} . \%$ latex dispersion will contain on the order of $10^{17}$ particles (200 $\mathrm{nm}$ diameter). Therefore, questions arise as to whether such images are truly representative of the entire bulk sample. Scattering techniques are very sensitive probes for samples with periodic structures. Detailed information about the interface between two polymer phases can be obtained with neutron scattering. However, sufficient contrast can usually only be achieved through the use of deuterium labelling.

NMR nuclear spin relaxation time analysis is an established method to detect the fractions of phases with different mobilities in a sample [10]. Furthermore, advanced NMR methods, such as field-gradient NMR diffusometry and solid-state NMR spindiffusion experiments, have been developed for the characterisation of heterogeneities in glassy polymers and polymer blends [11-14]. These techniques can be directly applied to a bulk sample without any modifications or preparation, such as thinning, cutting, coating, or labelling. An average measurement is obtained for a bulk sample. Therefore, the information extracted from these methods can be considered as a true representative of the initial bulk sample.

The larger the molecule's self diffusion coefficient $D(t)$ the greater the diffusion distance for a given time $t . D(t)=\left\langle r^{2}\right\rangle / 6 t$, where $\left\langle r^{2}\right\rangle$ is the mean square displacement and $t$ is diffusion time. If diffusion is restricted, as in a core of a core-shell particle or in porous media, then there will be less diffusive attenuation of the NMR signal for a given $t$. Thus, for $t$ much less than the time for a spin in a molecule to diffuse across a confinement or a pore, diffusion coefficients close to those of bulk material, $D_{b u l k}$, are measured, where as for longer $t$, the observed diffusion coefficient decreases as the molecules are increasingly restricted by the boundaries. This form the basis of microstructural investigations by NMR diffusometry.

Pulse field gradient (PFG) technique [15] has been widely used in the NMR diffusometry. This sort of diffusion experiments are usually discussed in terms of spin echoes, the amplitude of which is attenuated by translational displacements of the spin bearing molecules in the course of the pulse sequence that leads to determine $D(t)$. In the stimulated echo the effective diffusion time is prolonged by an intermittent pulse interval [15]. To measure very small $D(t)$ values using PFG method, large pulse gradients, that in practice difficult to achieve, are required. There are number of problems arising with pulse gradient experiments. Apart from fluctuations of strong and short gradient pulses in general, fields by eddy currents induced in metal components surrounding the probe must be compensated. Strong gradients stipulated strong currents through gradient coils which 
in tern may cause some thermal instability. These problems can safely be avoided by employing steady gradients. There are several advantages of steady gradient stimulated echo (SGSE) diffusometry such as no time dependent forces as in the PFG technique, stability is perfect and only limited by building vibrations, no time is lost by switching the gradients on and off, and achieving utmost possible echo attenuation due to the high field gradient. Usual SGSE methods contain two or three RF pulses [16]. The loss of the echo amplitude due to the relaxation is a major setback of these methods. Additionally, a separate signal must be measured as a reference, in the same experiment or from a separate experiment. A SGSE method, containing five RF pulses, compensating for relaxation losses has been presented by Kimmich [17]. The unique feature of this method is that it is a single scan/single-echo-signal type. That is, no reference signals - neither from the same pulse sequence nor from separate experiments - are needed.

Recent developments of NMR diffusometry have established its capability of measuring molecular self-diffusion coefficients down to values as low as $\sim 5 \times 10^{-15} \mathrm{~m}^{2} \mathrm{~s}^{-1}$, which is the physical limit for any diffusion measurements using NMR spin echoes $[11,12,17]$. Using the (SGSE) technique, Kimmich et al. measured the self-diffusion coefficient of linear, monodisperse poly(ethylene oxide) confined to nanoscopic pores of crosslinked methacrylate matrices. From their measurements of diffusion in confinement, they were able to deduce the tube diameter of polymer strands [12]. Diffusometry techniques are fundamentally different from microscopy techniques. Not only is NMR diffusometry capable of giving the average value for the sizes of phases in a two-phase composite, but it offers the unique ability to extract information on molecular dynamics in the same system at the same time.

Restricted diffusion refers to the influence posed by the spherical boundaries to the translational motion of molecules, which provides information on the size of a phase. It has been used elsewhere to measure the size of emulsion droplets [18,19]. But never has it been used to measure core of a core-shell structured polymer due to the difficulty in measuring the low diffusion coefficient $D(t)$ of the core material.

Herein, we report the application of five pulse SGSE method compensating for relaxation losses [17] to measure restricted diffusion in core-shell latex films For the first time, the average core size in a core-shell latex film is determined using the SGSE technique.

\section{MATERIALS AND METHODS}

Technical grade monomers methyl methacrylate (MMA) and butyl acrylate (BA), supplied by Quimidroga, and acrylic acid (AA, Aldrich) were used without purification. Divinylterminated polydimethylsiloxane (DV-PDMS, Aldrich), was used as resin; stearyl acrylate (SA, Aldrich) was used as a costabilizer; Dowfax 2A1 (alkyldiphenyl oxide disulfonate, Dow Chemical) was used as the surfactant. Potassium persulfate (KPS, Panreac) or a 1:1 molar ratio of ammonium persulfate (APS, Panreac) and sodium 
metabisulfite (SBS, Aldrich) was used as redox initiator system. Distilled water was used in all polymerizations. The high-solids-content (50 wt \% organic phase) silicone/acrylic hybrid latexes were synthesized by miniemulsion polymerization, in either batch or semicontinuous processes. The differences between the latexes is that they were prepared by batch miniemulsion polymerisation (BM25), semicontinuous batch miniemulsion polymerisation (SM25), and semicontinuous batch miniemulsion with a shot of monomer near the end of polymerisation (SBS25). The number in the code names for the latexes represents the mass of PDMS in grams per $100 \mathrm{~g}$ of acrylic monomer. More details of the materials preparation can be found elsewhere [20].

TEM images were obtained with a Hitachi 7000FA microscope. The latexes were stained by adding small amounts of phototungstic acid. The particles were cast on copper grids covered with polyvinylformal (Formvar R) and dried under a UV lamp in order to avoid film formation. Six different regions of the same sample were analyzed to ensure that the images were representative.

All NMR experiments were done in a small permanent magnet that has specifically designed pole pieces to give a magnetic field gradient of strength $17.5 \mathrm{~T} \mathrm{~m}^{-1}$ in the vertical direction. The details of this magnet, called Gradient at Right-angles to the field (GARField), have been reported [21] previously. Approximately $1 \mathrm{ml}$ of latex were cast on a clean, glass cover slip $(18 \mathrm{~mm} \times 18 \mathrm{~mm})$ and dried under ambient conditions. To ensure that all water was removed, the films were dried in vacuum for 6 hours at a temperature of $150^{\circ} \mathrm{C}$.

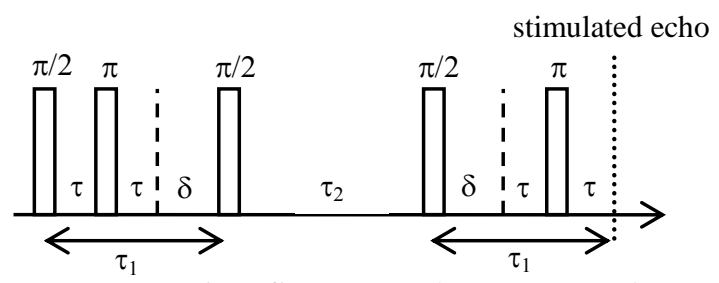

Figure 1: Pulse sequence consisting five RF pulses for steady gradient stimulated echo NMR diffusometry. Varying the interval $\delta$ while keeping $\tau_{1}$ and $\tau_{2}$ constant yields the attenuation curve of the stimulated echo for diffusion, whereas relaxation losses are constant.

The five pulse method, depicted in Fig. 1, was applied to obtain diffusion data from the PDMS chains in the core of polymer particles in the latex films. The dephased coherences are partially rephased in the first (constant) $\tau_{1}$ interval using a $\pi$ pulse. The remaining coherences are analogous to a pulse gradient stimulated echo (PGSE) experiment ${ }^{15}$ where two $\delta$ sections of the stationary gradient correspond to the gradient pulses. Diffusion experiments were carried out varying time $\delta$ but keeping the intervals $\tau_{1}$ and $\tau_{2}$ at constant values of $6.4 \mathrm{~ms}$ and $90 \mathrm{~ms}$, respectively. This meets the requirement $\tau_{2} \gg \tau_{1}$ for this type of experiment. The experiment measures non-restricted diffusion when $6 D_{b u l k} \tau_{1}<\left\langle\left\langle a^{2}\right\rangle\right.$ and restricted diffusion when $6 D_{b u l k} \tau_{2} \geq\left\langle a^{2}\right\rangle$, where $a$ is the size of the encapsulated region. More importantly all relaxation effects to the echo attenuation 
remain constant. Therefore, the decay of the stimulated echo amplitude $E(\delta)$ is determined solely by translational diffusion and it is given by [17]

$$
\ln E(\delta)=-\frac{1}{6} D \gamma^{2} G^{2}\left(\tau_{1}-\delta\right)^{3}-D \gamma^{2} G^{2} \delta^{2}\left(\tau_{2}+\frac{2}{3} \delta\right)
$$

where $\gamma$ is the gyromagnetic ratio, and $G$ is the field gradient strength. The first term in the Equation 1 corresponds to non-restricted diffusion of the spin bearing nuclei during and between two $\delta$ intervals and the second term corresponds to the restricted diffusion.

\section{RESULTS AND DISCUSSION}

TEM images of selected core-shell polymer particles of BM25 and SM25 are shown in Fig. 2. In these images, the dark component is attributed to PDMS and the lighter component is attributed to the acrylic copolymer. It can be seen that some of the polymer particles apparently do not have a PDMS core. Although the sample size is very small, it appears that there is a distribution of core sizes. The small number of particles in these images illustrates the difficulty in obtaining meaningful statistics on the distribution of core sizes. TEM images were not obtained on film cross-sections, so it is not known whether the particle structure is retained during film formation.

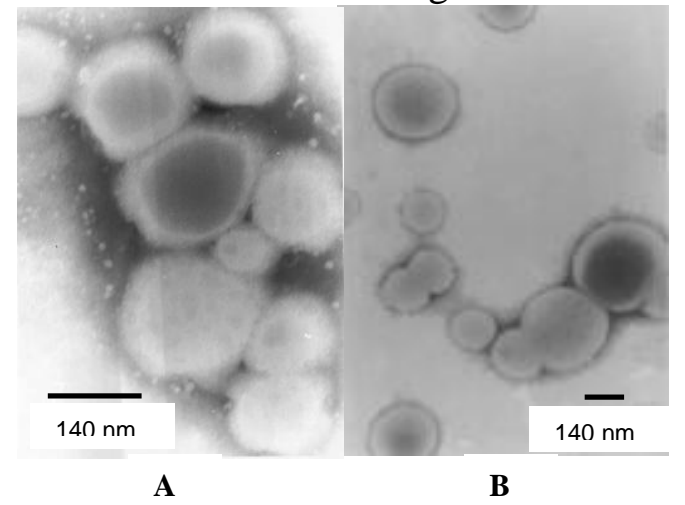

Figure 2: TEM images of core-shell latex particles: (A) BM25 and (B) SM25

The self diffusion coefficient of pure PDMS $D_{\text {bulk }}$ was calculated to be $7.3 \times 10^{-13}$ $\mathrm{m}^{2} \mathrm{~s}^{-1}$ in a separate experiment using the same source of PDMS as in the core-shell particles. Then the echo attenuation due to the non-restricted motion of the PDMS chains is calculated using the first term in Equation 1, wherein experimentally determined $D_{b u l k}$ for PDMS was used. The latter calculated attenuation was added to the original attenuation data so that the echo attenuation purely due to the restricted diffusion of PDMS is remained.

Experimental data were fitted to the exact solution for the attenuation due to the restricted diffusion at spherical boundaries found by Tanner and Stejskal: [22,23]

$$
E(\delta)=9[2 \pi q a \cos (2 \pi q a)-\sin (2 \pi q a)]^{2} /(2 \pi q a)^{6}
$$


where $a$ is the radius of a phase confined to a sphere and $q=\gamma G \delta$. In our experiments, $a$ represents the radius of the PDMS core.

Since the effect of the term $\gamma^{2} G^{2} \delta^{2}$ in the second part of Equation 1 is prominent compared to the rest of the term for the echo attenuation, and since the time durations $\tau_{1}$ and $\tau_{2}$ are kept at constant values, we plotted the experimental echo attenuation data against $\gamma^{2} G^{2} \delta^{2}$. Fig. 3 shows the experimental echo attenuation data obtained from the three core-shell polymer films (BM25, SBS25, and SM25), and the best fits to Equation 2. The open symbols in Fig. 3 represent the total echo attaenuation due to the unrestricted and restricted diffusion of PDMS in the core. It is not possible to fit the experimental data for a single core radius $a$ as the fitting parameter, which is consistent with the distribution of core sizes observed in the TEM sample. Instead, we obtained adequate fits to the data using two PDMS core radii and their corresponding volume fractions as the fitting parameters.

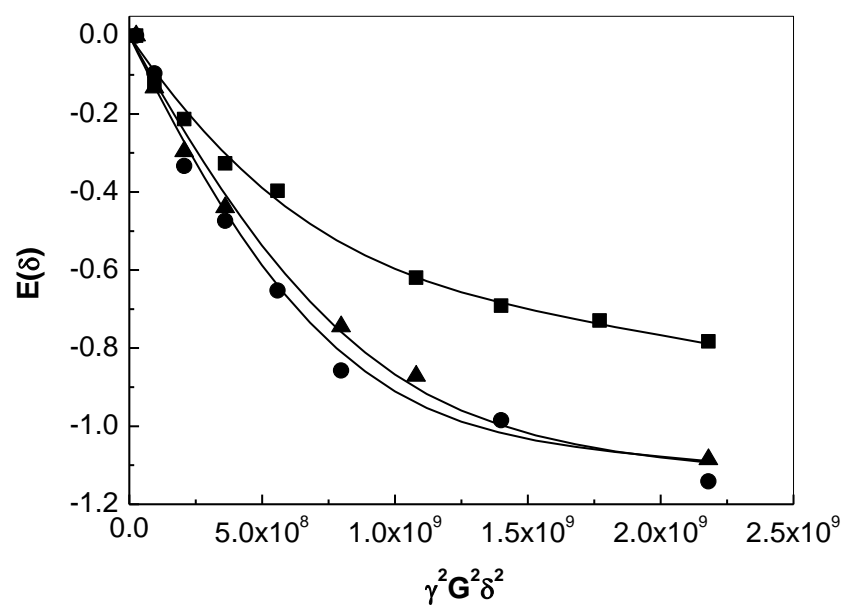

Figure 3: Normalised echo attenuation data for the latex films;

SM25, - - SBS25. Solid lines represent fit of the Equation 2 to experimental data. Open symbols represent data for total attenuation (i.e. nonrestricted + restricted)

The fact that there is evidence for confinement of the PDMS phase in the latex films indicates that the particle structure is retained in the films. There is no evidence for fracture of the acrylic shells. The particles are robust enough to resist coalescence and the coarsening of the phases. Instead, the nanostructure of the particles is used as a building block in the film.

NMR diffusometry reveals that the sizes of PDMS cores and their corresponding fractions are considerably different between BM25 and the other two latexes. The bestfits to the data reveal that BM25 contains $40 \%$ of $a=170 \mathrm{~nm}$ cores and $60 \%$ of $a=50 \mathrm{~nm}$ cores. On the other hand, SM25 and SBS25 both contain $60 \%$ of $a \approx 170 \mathrm{~nm}$ and $40 \%$ of $a \approx 40 \mathrm{~nm}$. 


\section{CONCLUSIONS}

The five-pulse NMR method has been employed for the first time to study restricted diffusion in core-shell latex films. This technique shows advantages in providing quantitative information on the bulk of a film, rather than relying on a small sample size. The results of our non-destructive experiment confirm that particle structure is retained in the film. There is no evidence for phase separation or segregation of PDMS cores. Moreover, differences in core sizes and corresponding fractions could convincingly be determined and correlated with the polymerisation techniques.

\section{Acknowledgement}

Funding for P.E. was provided by the European Commission's Sixth Framework Programme through the NAPOLEON Project (Contract No. IP 011844-2). R. Rodriquez, M.J. Barandiaran and J.M. Asua from POLYMAT, Spain are acknowledged for providing the samples.

\section{REFERENCES}

1. K. Landfester, C. Boeffel, M. Lambla, and H.W. Spiesss, Characterization of Interfaces in Core-shell Polymers by Advanced Solid-state NMR Methods, Macromolecules 29 (1996) 5972.

2. V.I. Eliseeva, Morphology and Phase Structure of Latex Particles and their Influence on the Properties of Latices and Films, Prog. Org. Coatings 13, (1985) 195.

3. M. Devon, J. Gordon, G. Roberts, and A. Rudin, Effects of Core-shell Latex Morphology on Film Forming Behavior, J. Appl. Polym. Sci. 39, (1990) 2119.

4. C. Schellenberg, K. Tauer, and M. Antonietti, Nano Sructured Polymer Films Based on Core-shell Latexes: Preparation and Characterization, Macromol. Symp. 151 (2000) 465.

5. S. Shen, M.S. El-Aaser, V. Dimonie, J.W. Vaderhoff, and E.D.Sudol, Preparation and Morphological Characterization of Microscopic Composite Particles, J. Polym. Sci., Polym. Chem. 29 (1991) 857.

6. S. Schellenberg, M. Akari, M. Regenbrecht, K. Tauer, F.M. Petrat, and M. Antonietti, Spherical Polymer Containers with a Fluid Polymer Core: Synthesis and Characterization of Film Formation by AFM, Langmuir 15, (1999) 1283.

7. L. Stapff, G. Weidemann, C. Schellenberg, M. Regenbrecht, M. Akari, and M. Antonietti, Tapping Mode Force Microscopy Imaging of Elastic Properties of Core-shellLlatex Particles, Surf. Interface. Anal. 27 (1999) 392.

8. N. Dingenouts, Y.S. Kimand, and M. Ballauff, The Interface Between Lmmiscible Polymers in Composite Latexes: a Small-Angle x-ray Scattering Study Employing Contrast Variation, Colloid. Polym. Sci. 272 (1994) 1380. 
9. W.D. Hergeth, H.J. Bittich, F. Eichborn, S. Schlenker, K. Schmutzler, and U.J. Steinau, Polymerizations in the presence of seeds: 5. Core-shell structure of two-stage emulsion polymers, Polymer $\underline{30}$ (1989) 1913.

10. C. Constantinuous, D. Tembou Nzudie, and G. Riess, NMR Nuclear Spin Relaxation Time Analysis to Detect the Fractions of Phases with Different Mobilities, Macromol. Chem. Rapid Commun. 24 (1993), 61.

11. E. Fischer, U. Beginn, N. Fatkullin, and R. Kimmich, Polymer Dynamics Under Nanoscopic Constraints: The "Corset Effect" as Revealed by NMR Relaxometry and Diffusometry, Mag. Res. Imag. 23 (2005) 379.

12. E. Fischer, and R. Kimmich, Constant Time Steady Gradient NMR Diffusometry Using the Secondary Stimulated Echo, J. Mag. Res. 166 (2004) 273.

13. E. Fischer, R. Kimmich, U. Beginn, M. Moeller, and N. Fatkulin, Segment Diffusion in Polymers Confined in Nanopores: A Fringe-Field NMR Diffusometry Study, Phys. Rev. E, $\underline{59}$ (1999) 4079.

14. K. Schmidt-Rohr, and H.W. Spiess, Multidimensional Solid State NMR and Polymers, (Academic, London, 1994).

15. C. Cho, Y. Hong, K. Kang, V.I. Volkov, V. Skirda, C.J. Lee, and C. Lee, Water Self-diffusion in Chlorella Studied by Pulse Field Gradient NMR, Mag. Res. Imag. $\underline{21}$ (2003) 1009.

16. R. Kimmich, W. Unrath, G. Schnur, and E. Rommel, NMR Measurement of Small Self-diffusion Coefficients in the Fringe Field of Superconducting Magnets, J. Mag. Res. 91 (1991) 136.

17. R. Kimmich, NMR Tomography Diffusometry Relaxometry, Springer-Verlag: Berlin Heidelberg, 1997.

18. P.J. McDonald, E. Ciampi, J.L. Keddie, M. Heidenreich, and R. Kimmich, Magnetic-Resonance Determination of the Spatial Dependence of the Droplet Size Distribution in the Cream Layer of Oil-in-water Emulsions: Evidence for the Effects of Depletion Flocculation, Phys. Rev. E 59 (1999) 874.

19. E. Liu, and K.M.McGrath, Emulsion Microstructure and Energy Input, Roles in Emulsion Stability, Coll. Surf. A $\underline{262}$ (2005) 101.

20. R. Rodriguez, C.J. Alarco'n, P. Ekanayake. P.J. McDonald, J.L. Keddie, M.J. Barandiaran, and J.M. Asua, Correlation of Silicone Incorporation into Hybrid Acrylic Coatings with the Resulting Hydrophobic and Thermal Properties, Macromolecules 41 (2008) 8537.

21. P.M. Glover, P.S. Aptaker, J.R. Bowler, E. Ciampi, and P.J. McDonald, A Novel High-Gradient Permanent Magnet for the Profiling of Planar Films and Coatings, J. Mag. Res. 139 (1999) 90.

22. E.O. Stejskal, and J.E. Tanner, Spin Diffusion Measurements: Spin Echoes in the Presence of a Time-dependent Field Gradient, J. Chem. Phys. $\underline{42}$ (1965) 288

23. J.E. Tanner and E.O. Stejskal, Restricted Self-diffusion of Protons in Colloidal Systems by the Pulsed-Gradient, Spin-echo Method, J. Chem. Phys. $\underline{49}$ (1968) 1768. 